\title{
ESTABLISHMENT OF A MODEL FOR INTERPRETATION AND CORRECTION TOOLS FOR BESSY II*
}

\author{
R. Bakker, D. Krämer, B. Kuske ${ }^{\dagger}$, P. Kuske, R. Müller, BESSY, Berlin, Germany
}

\begin{abstract}
From the very beginning of the commissioning of the Synchrotron Radiation facility BESSY II, work has been facilitated by online interpretation and correction procedures that utilise a commonly accessible online model of the storage ring optics. The software covers all linear properties like beta functions, orbit correction, tune variation etc. Initially the model has been set up from construction and alignment data and magnetic bench measurements. All relevant parameters are retrieved from a central reference database. During commissioning, the model has been adjusted in several steps, feeding back results from orbit response measurements. The achieved agreement is demonstrated.
\end{abstract}

\section{INTRODUCTION}

The BESSY II storage ring [1] has been commissioned during the last 9 months of 1998, and is in user-operation since the beginning of 1999. During 9 two-weeks machine development runs the reference optics has been established and the design parameters have been reached or surpassed [2].

The BESSY II control system [3] provides an online model of the storage ring optics, based on the GOEMON optics library [4]. Constant data, like the geometries and the conversion factors (current to magnetic field) which initially where extracted from magnetic bench measurements are taken from the ORACLE database. In combination with the actual currents read back from the power supplies, an online model is set up during the initialization phase of the orbit control software and the optic program and can be continuously updated. The calculated beta functions, tunes, chromaticities etc. can be displayed, and are used in correction algorithms, as e.g., the sensitivity matrices in the orbit correction. The correct translation from the power supply currents into the model was of essential importance for the progress during commissioning. Orbit response measurements (ORM) where used to get a better understanding of the storage ring hardware and improve the model.

\section{MODEL EVOLUTION DURING COMMISSIONING}

Already during the first run ORM were taken and revealed few hardware bugs, like a systematic polarity twist of all correction coils in the dipoles. Measurements taken during the second run, where the machine was run without sextupoles, led to a rise of $2-3 \%$ of the conversion factors

\footnotetext{
${ }^{*}$ Funded by the Bundesministerium für Bildung, Wissenschaft Forschung und Technologie and by the Land Berlin

† Email: kuskeb@bii.bessy.de
}

for the two defocusing quadrupole families located next to the dipoles. The initial difference between calculated and measured vertical tune could thus be fixed. The discrepancy to the bench measurements might be attributed to effects of the dipole edge, or to shielding effects. The conversion factors of the other Q-families were confirmed on the 1 percent level. The resulting orbit correction reached rms values of $\approx 1 \mathrm{~mm}$. During run 3, the sextupoles where switched on, and first measurements could be taken with a corrected orbit and better lifetimes. This led to a second adaption of the quadrupole conversion factors on the subpercent level. The resulting model was successfully used during the rest of the commissioning. The corrected orbit reached typical rms-values of $0.1 \mathrm{~mm}$ in both planes. The model has also been used to determine the locations of the BPMs with respect to the centers of the quadrupoles using beam based techniques [6]. The reference orbit going through the center of all 144 quadrupoles is accomplished to better than $0.15 \mathrm{~mm}$ in both planes. Beginning from run 7 , the linear optics started to settle, and 9 ORMs where at the same quadrupole settings, although the sextupoles, and definitely the steering coils were tuned differently. Only these measurements are presented in order to avoid the effect of different magnet settings and to outline the limits of an online model.

\section{ORBIT RESPONSE MEASUREMENTS}

The description and the analysis of ORMs has been published e.g., in [5]. Due to the positive experience at BESSY I and elsewhere, the measurement has been included as an automatic procedure in the control system, as well as the extraction of the sensitivity matrices from the measured data, since the beginning of the commissioning. Usually, the 64 horizontal and vertical steering coils included in the sextupoles are varied by $\pm 0.07 \mathrm{~A}$, while the weaker 16 horizontal coils in the dipoles are varied by $\pm 0.7 \mathrm{~A}$. Ten orbits are recorded for each setting of all steerers, where one orbit already represents a $100 \mathrm{~ms}$ average. The position error is $<1 \mu \mathrm{m}$ at any of the 112 BPMs [6]. This corresponds to an average error of the matrix elements, which are determined by linear regression, of $0.022 \mathrm{~mm} / \mathrm{A}$ in the horizontal and $0.017 \mathrm{~mm} / \mathrm{A}$ in the vertical plane. The quality of the horizontal data has been reduced by oscillations at multiples of $50 \mathrm{~Hz}$. However, the deviation between different measurements is large. It exceeds the accuracy of single measurements by more than an order of magnitude, even if the power supply settings of the ring only differ in the correction coil currents. These differences are attributed to differences in magnetic field history or drifts in temperature or current, or other parameters that have not been recorded. 
They complicate the extraction of a generally valid model.

\subsection{The Fitting Procedure}

The $\mathrm{K}$-values of the quadrupoles and the conversion factors of the corrector magnets were fitted to match the experimental conditions with the aid of the GOEMON tool. A singular value decomposition procedure has been used for the non-linear fit of the K-values while the conversion factors of the correction coils have been adjusted by a least square fit, iteratively. An 8-fold symmetric model has been utilised to determine the properties of the magnet families. Families equally powered and located in equivalent positions where fitted as one parameter, as the fit showed a tendency to differ between equivalent types of magnets without increasing the accuracy of the fit. The K-values of 7 types of quadrupoles and the conversion factors for 4 horizontal and 4 vertical types of corrector magnets were determined. Individual BPM sensitivities have not yet been included, as they could not be reliably extracted from the data. Malfunctioning BPMs where excluded from the data. Ambiguous BPMs could be determined during the fit, by comparing the measured and the calculated matrices Fig. 1. The rms deviation between the measured and the adjusted matrices was taken as indication of the quality of the approximation.

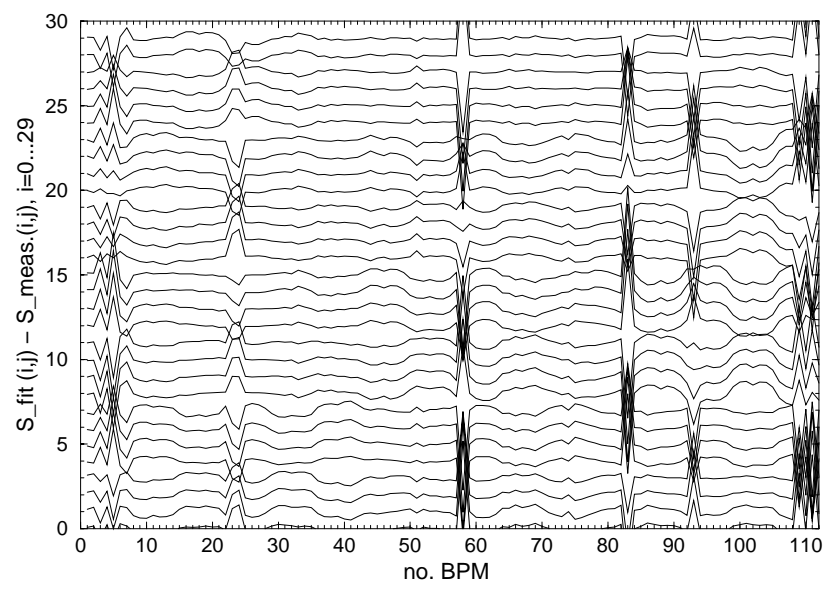

Figure 1: Malfunctioning BPMs show up as clear peaks in the difference between measured and fitted sensitivity matrices

\subsection{Results}

The resulting K-values and conversion factors of the 9 measurements taken for identical linear optics were compared and are displayed in figures 2 to 4 . The quality of the fit varies between 0.220 and $0.500 \mathrm{~mm} / \mathrm{A}$. Fig. 2 shows the relative deviation of the resulting $\mathrm{K}$-values to the averages for all families. The $\mathrm{K}$-values converge with increasing quality of the fit. For good fits $(\sigma<250 \mathrm{~mm} / \mathrm{A})$ they agree to better than 0.2 percent. No correlation with the settings of the sextupoles during the measurements could be detected, neither in degree of approximation nor in the scattering of the resulting K-values.

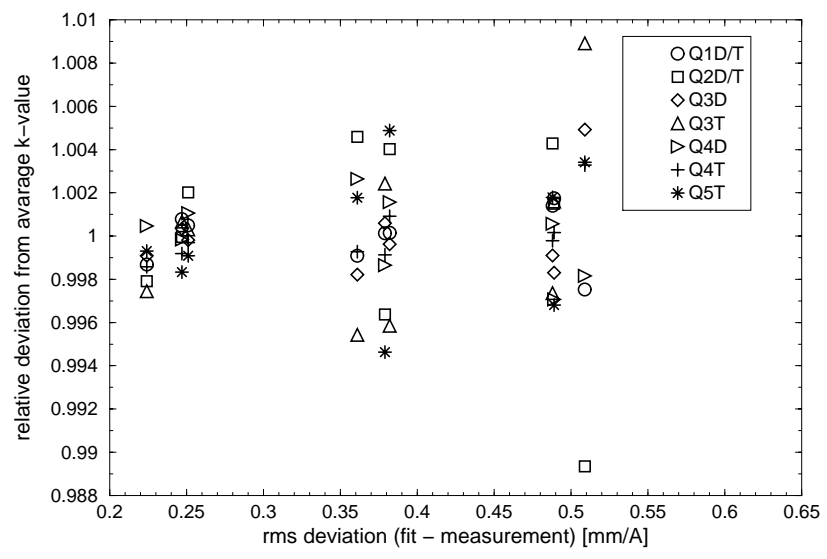

Figure 2: The resulting K-values from different runs agree to better than 0.2 percent for good fits

The resulting conversion factors for the steering coils are displayed in a similar way in Fig. 3. The deviation from the average value of all measurements is plotted over the quality of the fit. The spread is much wider than for the quadrupoles, and there is no correlation with the quality of the fit. Also in this case there was no correlation found between the settings of the sextupoles and the resulting conversion factors of the steerers included in the sextupole magnets.

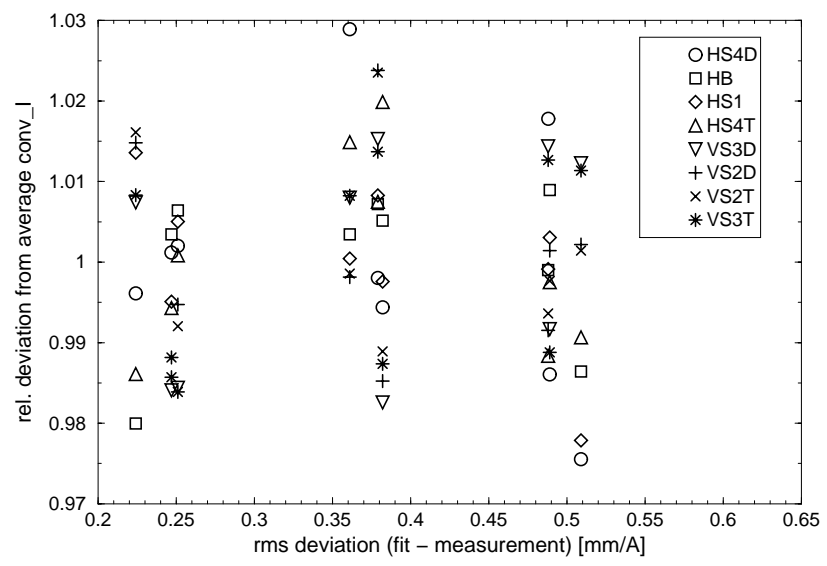

Figure 3: The conversion factors for the steering magnets show rms values of $\approx 1$ percent, independent of the quality of the fit

Fig. 4 shows the resulting tunes for all fits, the black symbols refer to the three best fits. For measurements where the working points have been recorded, there is a $10^{-4}$ agreement between the calculated and the measured tunes. Thus, the wide spread of tunes of almost 0.1 in both planes for different measurements taken for identical quadrupole currents does represent a real difference in the conditions during the measurement. The sextupole settings seem to correlate to some degree with the resulting tunes. Two fits show a clearly lower vertical tune (circle). Only in those measurements the chromatic sextupoles where reduced in current. Switching off the harmonic sextupoles 
(triangle) seems to cause a small rise in the both working points. But even measurements with identical sextupole settings (triangle) show a $\delta Q_{x} \approx 0.04$.

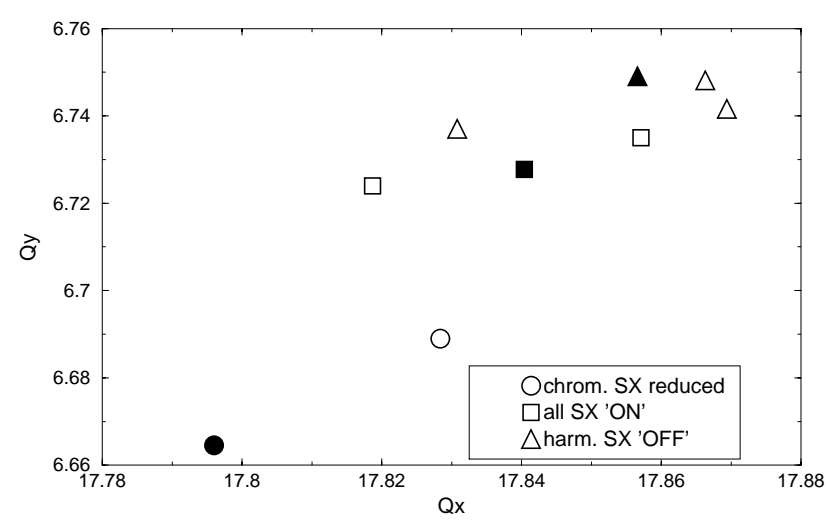

Figure 4: The tunes for all fits reveal a tune shift for cases where the chromatic sextupoles where reduced in current

\subsection{Interpretation}

The 8-fold symmetry of the ring can be used to average the effect of always eight equivalent steering elements, and create averaged, but symmetric sensitivity matrices. Comparing the averaged effects of the steerers with the original matrix, a rms deviation of $0.2-0.6 \mathrm{~mm} / \mathrm{A}$ was found. This varying degree of symmetry in the machine, just like the wide spread in tune and the difficulty to determine the steerer conversion factors indicate different (unretrievable) machine conditions during the measurements. It is expected that these differences will diminish for a machine in full operation. So far, it does limit the achievable accuracy of the model. There is a clear correlation between the accuracy reached in the fit and the degree of divergence of single steerers from the average. This correlation indicates that even for reproducible machine conditions, the accuracy of the fit can be only improved by splitting up the magnet families and allowing individual conversion factors. According to the bench measurements the deviation of single quadrupoles from their families average is of the order of a few per mill. Out of the 3 best fits $(\sigma<0.25$ $\mathrm{mm} / \mathrm{A}$ ), only one measurement utilises the regular settings of all sextupoles, and it was taken to set up the new model. Fig. 5 shows the very satisfactory agreement between the beta functions calculated from the new model and those determined by local variation of the quadrupole fields.

\subsection{Latest Results}

During the last machine shift, (March 1999), the oscillation of the horizontal beam position could be considerably reduced, and the quadrupoles have been slightely adjusted to achieve more symmetrical beta functions. Three ORM have been performed during 2 days and show a good reproducibility in tune of $10^{-4}$. The fitted K-values agree to better than 0.1 percent, but there are reproducible differences to up to 0.5 percent to the K-values of the new model

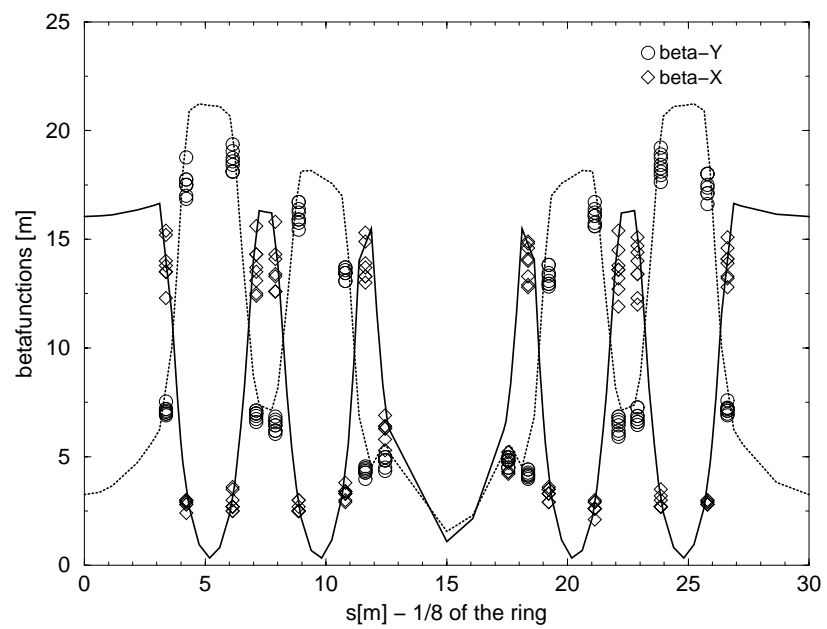

Figure 5: The measurement of local beta functions agrees well with the new model

extracted from the last measurements, which might be due to the optics changes.

\section{CONCLUSION}

The utilisation of an online model in combination with the very early realization of ORMs has proven very fruitful during the BESSY II commissioning. In two steps a quality was reached that satisfied commissioning needs. For a more detailed analysis, especially effects of single magnets, the optics has to be finally settled and the conditions during the ORMs have to be recorded more carefully in order to isolate the effects that lead to the bad consistency of the extracted sensitivity matrices. The model has to be extended from magnet families to individual magnets in order to improve the approximation to be comparable to the accuracy of the measurements. With increasing confidence in single measurements also the individual sensitivity of the BPMs has to be included in the model.

\section{REFERENCES}

[1] E. Jaeschke et al., "Lattice Design for the $1.7 \mathrm{GeV}$ Light Source BESSY II", IEEE Part. Acc. Conf. Washington, (1993), p 1474.

[2] R. Bakker et al., "Status and Commissioning Results of BESSY II", this conference.

[3] R. Müller et al., "Rapidly Installable High Performance Control System Facilitates BESSY II Commissioning", EPAC Stockholm (1998), p 1676.

[4] H. Nishimura, "Object-oriented software construction at ALS", Nuclear Instruments and Methods in Physics Research A 352 (1994) 379-382.

[5] J. Safranek, "Experimental Determination of Storage Ring Optics using Orbit Response measurements", Nuclear Instruments and Methods in Physics Research A 388 (1997) 27-36.

[6] P. Kuske et al., "Experience with the Beam Position Monitor System of the BESSY II storage Ring", this conference. 